\title{
APIGENIN RESTORES NORMAL VASCULAR REACTIVITY IN DIABETIC RATS VIA PROTEIN KINASE C INHIBITION
}

\author{
Nora Desoky ${ }^{1}$, Hany M. El-Bassossy ${ }^{1,2}$, Ahmed Fahmy ${ }^{1}$ and Ahmad Azhar ${ }^{3}$ \\ ${ }^{1}$ Department of Pharmacology, Faculty of Pharmacy, Zagazig University, Egypt \\ ${ }^{2}$ Department of Pharmacology, Faculty of Pharmacy, King Abdulaziz University, kingdom of Saudi Arabia \\ ${ }^{3}$ Department of Pediatric Cardiology, Faculty of Medicine, King Abdulaziz University, kingdom of Saudi Arabia
}

\begin{abstract}
Deterioration of vascular reactivity has pivotal role in diabetic vascular complications. Apigenin is a natural flavonoid with protein kinase $\mathrm{C}$ inhibiting activity and antioxidant effect.

Objective: In the present study, the effect of the flavonoid apigenin on diabetes-induced deteriorated vascular reactivity was investigated.

Materials and methods:Insulin deficiency was induced by streptozotocin while, insulin resistance (IR) by fructose. Rats were left tenor twelve weeks after STZ or fructose administration respectively, isolated thoracic aorta reactivity to phenylephrine (PE), $\mathrm{KCl}$, acetylcholine (ACh) and sodium nitroprusside (SNP) was studied.

Results: Insulin deficiency increased responses to $\mathrm{PE}$ and $\mathrm{KCl}$, decreased response to $\mathrm{ACh}$ but not affect response to SNP, while incubation with apigenin $(7 \mu \mathrm{M}, 20 \mathrm{~min})$ normalized responses to $\mathrm{PE}, \mathrm{KCl}$ and ACh. In addition, insulin resistance (IR) increased responses to $\mathrm{PE}$ and $\mathrm{KCl}$, decreased response to $\mathrm{ACh}$ but not affect the response to SNP, while in vitro incubation with apigenin normalized responses to $\mathrm{PE}, \mathrm{KCl}$ and $\mathrm{ACh}$. Furthermore, , protein kinase $\mathrm{C}$ stimulation by incubation with phorbol 12-myristate 13-acetate (PMA, $800 \mathrm{nM}, 1 \mathrm{~h}$ ) led to a similar impairment in isolated normal aorta to that seen in case of insulin deficiency and insulin resistance while co-incubation with apigenin restored normal vascular reactivity.

Discussion and conclusion: Apigenin, a natural protein kinase $\mathrm{C}$ inhibitor, restores normal vascular reactivity in diabetes via a mechanism involving PKC inhibition.

Key words: Diabetes; insulin resistance, aorta; contraction, relaxation; apigenin.

Abbreviations: ANOVA, analysis of variance; NO, nitric oxide; PE, phenylephrine; ROS, reactive oxygen species; SNP, sodium nitroprusside; PMA, phorbol 12-myristate 13-acetate;PKC, protein kinase C
\end{abstract}

\section{INTRODUCTION}

D iabetes is a disorder caused by insulin deficiency or resistance. Insulin resistance is a component of glucose intolerance [1], which is compensated by prolonged hyperinsulinemia. Eventually, pancreatic $\beta$-cells fail to secrete sufficient insulin, leading to non-insulin-dependent diabetes mellitus [2].

In spite of the significant developments in antidiabetic therapy, diabetic complications continue to be seriously deleterious. Various types of diabetic complications affecting different systems in the body have been reported. The clinical course of the disease is largely determined by those complications. This is why prevention or treatment of the disabling complications associated with diabetes is very important [3]. Many of the clinical complications of diabetes may be ascribed to alterations in vascular structure and function, with subsequent end-organ damage and death [4]. Therefore, preventing vascular complications in diabetes can save diabetic patient from other complications.

Flavonoids are diphenylpropanoids that occur far and wide in plant foods and form important constituents of human diet. Apigenin is a 4',5,7 trihydroxy flavone, belongs to a less toxic and nonmutagenic [5] flavone subclass of flavonoids. It is ubiquitously distributed in leaves, vegetables, stems and fruits of several vascular plants. It has been shown to act as a potent antioxidant[6], protein kinase $\mathrm{C}$ inhibitor[6-8]. Apigenin is a flavone rich in the Chinese herb FlosChrysanthemi and many other plants. It has a variety of pharmacological activities, including hypotensive [9], It has been reported that extract of FlosChrysanthemi caused vasodilatation in rat thoracic aorta [10]. However, the effects of apigenin on aortaeisolated from diabetic animals have not been clarified in isolated vascular preparations.

Therefore, the aim of the present work was to examine the effect of apigenin on diabetes-induced deteriorated vascular reactivity and illustrate its mechanism of action.

\subsection{Animals}

\section{METHODS}

Adult male Wistar albino rats with starting weight of 140-160 $\mathrm{g}$ for the insulin deficiency part and 90-110 g for insulin resistance part (Zagazig University, Zagazig, Egypt) were housed in standard polypropylene cages (five rats per cage) and kept on a light-dark cycle of equal duration, 
under constant environmental conditions. Rats were fed commercially available rat normal pellet diet and water ad libitum. Experimental design and animal handling were accordingto the guidelines of the Ethical Committee of the Facultyof Pharmacy, Zagazig University, for Animal Use.

2.2 Study protocol

Two animals models were used; insulin deficiency and insulin resistance. In each model, animals were randomly divided into fourexperimental groups (8 animals each); control, insulin deficient (ID) or insulin resistant (IR), apigenin-ID or apigenin-IR and apigenin-control. Insulin deficiency was induced by a single intraperitoneal injection of streptozotocin (STZ, 50 $\left.\mathrm{mg} \cdot \mathrm{kg}^{-1}\right)$. Insulin resistance was induced by adding fructose $(10 \%)$ to the drinking water. Rats were left 10 weeks or 12 weeks after STZ or fructose administration respectively.

At the end of the study, through opening the abdomen, descending thoracic aorta was carefully excised and placed in a Petri dish filled with cold Krebs-Henseleit buffer containing (in $\mathrm{mM}$ ): $\mathrm{NaCl}$ 118.1, $\mathrm{KCl}$ 4.69, $\mathrm{KH}_{2} \mathrm{PO}_{4} 1.2, \mathrm{NaHCO}_{3}$ 25.0, glucose 11.7, $\mathrm{MgSO}_{4} 0.5$ and $\mathrm{CaCl}_{2} 2.5$. The aorta was cleaned of excess connective tissue and fat and cut into rings of approximately $3 \mathrm{~mm}$ in length for studying vascular reactivity. In some experiments, aortic rings from control, insulin deficient or insulin resistant animals were incubated with apigenin $(7 \mu \mathrm{M}, 20 \mathrm{~min})$ beforestudying vascular reactivity. The incubation concentration and time of apigenin was chosen based upon that was previously reported[11] which was shown to significantly inhibit protein kinase $\mathrm{C}$.

2.3V ascular reactivity

The vascular reactivity to various vasoconstrictors and vasodilators was investigated as early described [12;13]. Thoracic aorta rings were suspended under $8 \mathrm{mN}$ resting tension in individual $30 \mathrm{ml}$ organ chambers containing KrebsHenseleit buffer at $37^{\circ} \mathrm{C}$ and aerated with $95 \%$ oxygen, $5 \%$ carbon dioxide. Ring tension was determined by use of an isometric force transducer (Biegestab K30, HugosachsElektronik, March, Germany). Force displacement was recorded with a PowerLab Data Interface Module connected to a PC running Chart software (v4.2, ADI Instruments, Chalgrove, Oxon, UK). Rings were equilibrated for 60 min during which time, the bath solution was changed every $30 \mathrm{~min}$. Before beginning the experiment, vessel viability was assessed by exposing arteries to $\mathrm{KCl}(80 \mathrm{mM})$. This was repeated until stable responses were achieved (usually two exposures). For studying the vasoconstrictor responsiveness of aorta, cumulative concentrations of phenylephrine (PE, $10^{-9}$ to $10^{-5} \mathrm{M}$ ) or $\mathrm{KCl}(10$ to $100 \mathrm{mM}$ ) were added to the organ bath and the response was recorded. For studying the vasodilator responsiveness of aorta, rings were first precontracted with submaximal concentrations of PE $\left(10^{-6}\right.$ in case of control or $3 \times 10^{-7}$ in case of insulin deficient or resistant group). The cumulative concentrations of acetylcholine (ACh, $10^{-9}$ to $10^{-5}$ $\mathrm{M})$ or sodium nitroprusside ( $\mathrm{SNP}, 10^{-9}$ to $10^{-5} \mathrm{M}$ ) were then added to the organ bath and the response was recorded. The submaximal concentration of PE was chosen to give similar precontraction in all studied groups. For studying the effect of PMA and apigenin on vascular reactivity, PMA $(800 \mathrm{nM})$ and apigenin $(7 \mu \mathrm{M})$ were included in the organ bath during the 60 min equilibration period and during the addition of vasoactive agents.

2.4 Drugs and chemicals

The following drugs and chemicals were used: Fructose (El-Nasr Chemical Co., Cairo, Egypt); STZ, apigenin, PMA, ACh, PE, SNP (SigmaAldrich, Dorset, UK). Apigenin was dissolved in $0.5 \mathrm{~N} \mathrm{NaOH}$ and PMA was frist dissolved in DMSO and diluted 10 times in Krebs Henseleit buffer. ACh, PE and SNP were dissolved in distilled water.

2.5 Statistical analysis

All data are expressed as mean \pm SEM. Statistical analysis was performed by the analysis of variance (ANOVA) followed by Newman-Keuls' post hoc test. The agonist maximum response $\left(\mathrm{E}_{\max }\right)$ was calculated from concentration-response curve by non-linear regression analysis of the curve using computer based fitting program and used for comparison (Prism 5, Graphpad, CA, USA).

\section{RESULTS}

3.1. Blood glucose, insulin and pressure Intra-peritoneal administration of STZ in a dose of $50 \mathrm{mg} \mathrm{kg}{ }^{-1}$ led to a significant elevation in blood glucose $(p<0.05)$ and a significant decrease in serum insulin $(\mathrm{p}<0.001)$ compared to control values. The STZ-induced insulin deficient rats had a significantly elevated systolic $(\mathrm{p}<0.001)$ and diastolic BP ( $\mathrm{p}<0.001)$ compared to control (table $1)$.

Addition of fructose to the drinking water in concentration of $10 \%$ for 12 weeks led to a significant elevation in blood glucose $(\mathrm{p}<0.001)$ and 
serum insulin $(\mathrm{p}<0.001)$ compared to control values. The fructose-induced insulin resistant rats had a significantly elevated systolic $(p<0.001)$ and diastolic BP ( $\mathrm{p}<0.001$ ) compared to control (table 2).

3.2 Vascular reactivity

Cumulative addition of PE (PE, $10^{-9}$ to $10^{-5} \mathrm{M}$ ) or $\mathrm{KCl}\left(10^{-2}\right.$ to $\left.10^{-1} \mathrm{M}\right)$ to the organ bath resulted in concentration dependent contraction of aorta in all the groups. Insulin deficiency induced by STZ resulted in anincrease in aorta responsiveness to PE, reflected by a significant increase in the apparent $\mathrm{E}_{\max }(\mathrm{p}<0.001$, Fig. 1a and table 3$)$ and to $\mathrm{KCl}$, reflected by a significant increase in the apparent $\mathrm{E}_{\max }(\mathrm{p}<0.001$, Fig. $1 \mathrm{~b}$ and table 3).Apigenin (AP, $7 \mu \mathrm{M}, 1 \mathrm{~h})$ incubation prevented the insulin deficiency-induced hyper responsiveness to PE, reflected by a significant increase in apparent $E_{\max }(\mathrm{p}<0.001$, Fig. 1a and table 3)and the hyperresponsiveness to $\mathrm{KCl}$, reflected by a significant increase in apparent $E_{\max }(p<0.001$, Fig. $1 b$ and table $3)$.

Cumulative addition of $\mathrm{ACh}\left(10^{-9}\right.$ to $\left.10^{-5} \mathrm{M}\right)$ to the organ bath resulted in concentration-related decreases in the tension of aortic artery rings precontracted with phenylephrine. Insulin deficiency led to a large decrease in aorta responsiveness to $\mathrm{ACh}$, reflected by a significant decrease in apparent $E_{\max }(p<0.001$, Fig. 1c and table 3). AP protected against insulin deficiencyinduced ACh hypo-responsiveness, reflected by a significant decrease in apparent $\mathrm{E}_{\max }$ compared with insulin deficient group $(\mathrm{p}<0.001$, Fig. $1 \mathrm{c}$ and table 3). However, neither insulin deficiency nor AP treatment affect isolated aorta responsiveness to SNP(Fig. 1d and table 3).

The insulin resistance rats showed a large increase in aorta responsiveness to both $\mathrm{PE}$, reflected by a significant increase in apparent $\mathrm{E}_{\max }(\mathrm{p}<0.001$, Fig. $2 \mathrm{a}$ and table 4 ) and to $\mathrm{KCl}$, reflected by a significant increase in apparent $E_{\max }(p<0.001$, Fig. $2 b$ and table 4). While, AP incubation significantly inhibited it the insulin resistance-induced hyper responsiveness to $\mathrm{PE}$ and to $\mathrm{KCl}$ (both at $\mathrm{p}<0.001$, Fig. 2a\&b, table 4). On the other hand, insulin resistance led to a decrease in aorta responsiveness to $\mathrm{ACh}$, reflected by a significant decrease in apparent $\mathrm{E}_{\max }(\mathrm{p}<0.001$, Fig. 2c, table 4).AP incubation prevented the insulin resistance-induced ACh hypo-responsiveness $(\mathrm{p}<0.001$, Fig. $2 \mathrm{c}$, table 4). However, neither insulin resistance nor AP treatment affect isolated aorta responsiveness to SNP (Fig. 2d and table 4).

Incubation of aorta with PMA $(800 \mathrm{nM}, 1 \mathrm{~h})$ or AP $(7 \mu \mathrm{M}, 1 \mathrm{~h})$ had no effect on the basal tension. In contrast, incubation with PMA (800nM, 1h) resulted in a large increase in aorta responsiveness to $\mathrm{PE}$ and $\mathrm{KCl}$, reflected by a significant increase in apparent $\mathrm{E}_{\max }$ (both at $\mathrm{P}<0.001$, Fig $3 \mathrm{a}$ and $3 \mathrm{~b}$, table 5)However, incubation with AP $(7 \mu \mathrm{M}, 1 \mathrm{~h})$ protected against PMA-induced hyperresponsiveness to $\mathrm{PE}$ or $\mathrm{KCl}$ (both at $\mathrm{p}<0.001$, Fig $3 \mathrm{a}$ and 3b, table 5). PMA and apigenin incubation led to no significant change in aorta responsiveness to $\mathrm{ACh}$, (data not shown). AP incubation did not change aorta responsiveness to any of the used vasoactive agents compared to control (Fig 1-3, tables 3-5).

\section{DISCUSSION}

The purpose of this study was to investigate the effect of apigenin against diabetes-induced deterioration in vascular reactivity. We have shown for the first time that acute in vitro incubation with apigenin restored normal vascular reactivity in thoracic aorta isolated from either insulin deficient or insulin resistance animals. In addition, acute in vitro incubation with apigenin protected from exaggerated vasoconstriction responses induced by PKC stimulation. These findings suggest a novel biological activity for the well tolerated natural flavonoid, apigenin in the management of diabetic vascular complications.

As, diabetes is a disorder caused by insulin deficiency or resistance which are completely distinguished in characters, we investigated apigenin effect in two well established models of diabetes. In streptozotocin (STZ) model, single dose of STZ (50 mg. $\mathrm{kg}^{-1}$, ip) developed significant hyperglycemia but hypoinsulinemia in 8 weeks. streptozotocin has been widely used to induce insulin deficiency through pancreatic $\beta$-cell destruction [14]. In the fructose model, including fructose $(10 \%)$ in drinking water developed significant hyperinsulinemia and mild hyperglycemia in 12 weeks. This appeared to be most suitable for production of fructose-induced insulin resistance [15].

In this study, insulin deficiency was associated with deterioration in vascular reactivity. We have found significant increases in aorta responsiveness to PE and to $\mathrm{KCl}$ and a large decrease in aorta responsiveness to ACh. However, insulin deficiency did not affect isolated aorta responsiveness to SNP. 
Hyperglycemia due to insulin deficiency has been reported to cause vascular abnormalities, such as increase in the response to vasoconstrictor agents $[16 ; 17]$ and an impairment of endotheliumdependent vasodilatation [18].In the insulin resistance model, we have reported an increased contraction of aorta to both $\mathrm{PE}$ and to $\mathrm{KCl}$ and a decrease in aorta responsiveness to ACh. However, insulin resistance did not affect isolated aorta responsiveness to SNP. There is an increasing evidence that, insulin resistance has adverse effects on the reactivity of arteries and arterioles and promotes arterial hypertension and vascular occlusive diseases [2]. Thoracic aorta from fructosefed rats showed exaggerated responses to $\mathrm{PE}$ and $\mathrm{KCl}[19 ; 20]$ while several studies have shown that fructose-fed rats exhibit impaired endotheliumdependent relaxation in response to ACh [21]. Similar findings concerning impairment of endothelium-dependent dilation in insulin resistance people have been derived from several regional circulations [22].The observed exaggerated vasoconstriction response could be due to increased extracellular calcium influx [16]. Instead, contractile responses can be modulated by agonists independently of changes in intracellular $\mathrm{Ca}^{2+}$ through inhibition of myosin light chain phosphatase activity due to protein kinase $\mathrm{C}$ (PKC) activation, a process known as $\mathrm{Ca}^{2+}$ sensitization $[23 ; 24]$. On the other hand, the impairment in endothelial dependent relaxation could be through inhibition of NO generation. We have shown previously impairment in pulmonary artery endothelial dependent relaxation which was mediated by stimulation of reactive oxygen species (ROS) generation and nitric oxide synthase uncoupling [25].

The present study shows that $20 \mathrm{~min}$ in vitro incubation with apigenin $(7 \mu \mathrm{M})$ was able to restore normal vascular reactivity in aorta isolated from both insulin deficient or resistant animals. As, apigenin significantly inhibited the hyperresponsiveness to both $\mathrm{PE}$ and $\mathrm{KCl}$ while protected from ACh hypo-responsiveness in aorta isolated from both insulin deficient and resistant animals. The direct effect of apigenin on exaggerated vascular contractility associated with diabetes is seems to be mediated at least in part by PKC inhibition as apigenin is used in this study at the concentration it was reported to inhibit PKC[11]. In support of this hypothesis, we have found that incubation with PMA $(800 \mathrm{nM}, 1 \mathrm{~h})$ resulted in a similar increase in aorta responsiveness to PE and $\mathrm{KCl}$ to that seen in insulin deficiency or resistance. However, incubation with apigenin protected against PMA-induced hyperresponsiveness to PE or $\mathrm{KCl}$. On the other hand, the effect of apigenin on impaired vasodilatation in diabetes is likely mediated by its antioxidant effect. Apigenin is a well-known potent antioxidant[6]. Suppression of ROS by apigenin saves NO from quenching and hence restores normal vascular dilatation.

In conclusion, apigenin restored normal vascular reactivity in thoracic aorta isolated from either insulin deficient or insulin resistance animals by mechanisms involving protein kinase $\mathrm{C}$ inhibition.

\section{DECLARATION OF INTEREST}

This work is funded by a research grant ID1024 provided by the Science and Technology Development Fund, Ministry of Higher Education, Egypt. STDF did not have any role in in study design; in the collection, analysis or interpretation of data; in the writing of the report; or in the decision to submit the paper for publication. No other funding source and there is no conflict of interest.

\section{REFERENCES}

1. Donnelly R and Qu X, Mechanisms of insulin resistance and new pharmacological approaches to metabolism and diabetic complications. Clin Exp Pharmacol Physiol 1998;25:79-87.

2. Busija DW, Miller AW, Katakam P, Erdos B, Adverse effects of reactive oxygen species on vascular reactivity in insulin resistance. Antioxid Redox Signal 2006;8:1131-40.

3. Altan VM, The pharmacology of diabetic complications. Curr Med Chem 2003;10:131727.

4. Akbari CM and LoGerfo FW, Diabetes and peripheral vascular disease. J Vasc Surg 1999;30:373-84.

5. Hertog MG, Intake of potentially anticarcinogenic flavonoids and their determinants in adults in The Netherlands 1993.

6. Nielsen SE, Effect of parsley (Petroselinum crispum) intake on urinary apigenin excretion, blood antioxidant enzymes and biomarkers for oxidative stress in human subjects 1999.

7. Nielsen SE, Effect of parsley (Petroselinum crispum) intake on urinary apigenin excretion, blood antioxidant enzymes and biomarkers for oxidative stress in human subjects 1999.

8. Lin JK, Suppression of protein kinase $\mathrm{C}$ and nuclear oncogene expression as possible molecular mechanisms of cancer 
chemoprevention by apigenin and curcumin 1997.

9. Loizzo MR, Said A, Tundis R, Rashed K, Statti GA, Hufner A, Menichini F, Inhibition of angiotensin converting enzyme (ACE) by flavonoids isolated from Ailanthus excelsa (Roxb) (Simaroubaceae). Phytother Res 2007;21:32-6.

10. Jiang HD, Cai J, Xu JH, Zhou XM, Xia Q, Endothelium-dependent and direct relaxation induced by ethyl acetate extract from Flos Chrysanthemi in rat thoracic aorta. J Ethnopharmacol 2005;101:221-6.

11. Zhang YH, Park YS, Kim TJ, Fang LH, Ahn HY, Hong JT, Kim Y, Lee CK, Yun YP, Endothelium-dependent vasorelaxant and antiproliferative effects of apigenin. Gen Pharmacol 2000;35:341-7.

12. El-Bassossy HM, El-Moselhy MA, Mahmoud MF, Pentoxifylline alleviates vascular impairment in insulin resistance via TNF-? inhibition. Naunyn Schmiedebergs Arch Pharmacol 2011;384:277-85.

13. Mahmoud MF, El-Nagar M, El-Bassossy HM, Anti-inflammatory effect of atorvastatin on vascular reactivity and insulin resistance in fructose fed rats. Arch Pharm Res 2012;35:15562.

14. Hu N, Xie S, Liu L, Wang X, Pan X, Chen G, Zhang L, Liu H, Liu X, Liu X, Xie L, Wang G, Opposite Effect of Diabetes Mellitus Induced by Streptozotocin on Oral and Intravenous Pharmacokinetics of Verapamil in Rats. Drug Metab Dispos 2011;39:419-25.

15. Dai $\mathrm{S}$ and McNeill JH, Fructose-induced hypertension in rats is concentration- and duration-dependent. $\mathbf{J}$ Pharmacol Toxicol Methods 1995;33:101-7.

16. Xavier FE, Davel AP, Rossoni LV, Vassallo DV, Time-dependent hyperreactivity to phenylephrine in aorta from untreated diabetic rats: role of prostanoids and calcium mobilization. Vascular Pharmacology 2003;40:67-76.

17. Kinoshita Y, Saito M, Satoh I, Shomori K, Suzuki H, Yamada M, Kono T, Satoh K,
General administration of cyclohexenonic longchain fatty alcohol ameliorates hyperreactivity of STZ-induced diabetic rat aorta. Life Sciences 2006;78:1508-14.

18. Baluchnejadmojarad $\mathrm{T}$, Roghani $\mathrm{M}$, Homayounfar H, Hosseini M, Beneficial effect of aqueous garlic extract on the vascular reactivity of streptozotocin-diabetic rats. Journal of Ethnopharmacology 2003;85:139-44.

19. Iyer SN and Katovich MJ, Vascular reactivity to phenylephrine and angiotensin II in hypertensive rats associated with insulin resistance. Clin Exp Hypertens 1996;18:227-42.

20. Shinozaki K, Ayajiki K, Nishio Y, Sugaya T, Kashiwagi A, Okamura T, Evidence for a Causal Role of the Renin-Angiotensin System in Vascular Dysfunction Associated With Insulin Resistance. Hypertension 2004;43:255-62.

21. Takagawa Y, Berger ME, Hori MT, Tuck ML, Golub MS, Long-term fructose feeding impairs vascular relaxation in rat mesenteric arteries. Am J Hypertens 2001;14:811-7.

22. Carmassi F, De Negri F, Fioriti R, De Giorgi A, Giannarelli C, Fruzzetti F, Pedrinelli R, Dell'Omo G, Bersi C, Insulin resistance causes impaired vasodilation and hypofibrinolysis in young women with polycystic ovary syndrome. Thromb Res 2005;116:207-14.

23. Morgan JP and Morgan KG, Calcium and cardiovascular function. Intracellular calcium levels during contraction and relaxation of mammalian cardiac and vascular smooth muscle as detected with aequorin. Am J Med 1984;77:33-46.

24. Somlyo AP and Somlyo AV, Signal transduction by G-proteins, Rho-kinase and protein phosphatase to smooth muscle and nonmuscle myosin II. J Physiol 2000;522:177-85.

25. El-Bassossy HM, El-Maraghy NN, El-Fayoumi HM, Watson ML, Haem oxygenase-1 induction protects against tumour necrosis factor alpha impairment of endothelial-dependent relaxation in rat isolated pulmonary artery. $\mathrm{Br} \mathrm{J}$ Pharmacol 2009;158:1527-35. 